\title{
FAST SIMULATION OF TEMPRATURE AND GRAIN GROWTH IN DIRECTED ENERGY DEPOSITION ADDITIVE MANUFACTURING
}

\author{
D. WEISZ-PATRAULT ${ }^{1}$, S. SAKOUT ${ }^{2}$ AND A. EHRLACHER ${ }^{3}$ \\ ${ }^{1}$ LMS, CNRS, École Polytechnique, Institut Polytechnique de Paris, F-91128 Palaiseau, France, \\ daniel.weisz-patrault@polytechnique.edu \\ ${ }^{2}$ LMS, CNRS, École Polytechnique, Institut Polytechnique de Paris, F-91128 Palaiseau, France, \\ sofia.sakout@polytechnique.edu \\ ${ }^{3}$ Navier, CNRS, École des Ponts ParisTech, 6 \& 8 Ave Blaise Pascal, F-77455 Marne La Vallee, \\ France, alain.ehrlacher@enpc.fr
}

Key words: Directed Energy Deposition, Thermal cycling, Grain growth, Fast modeling

\begin{abstract}
In this paper, a fast simulation of grain growth during directed energy deposition is presented. Controlling the microstructure is indeed essential to obtain the desired macroscopic behavior. We present a fast macroscopic simulation of temperature accounting for grain growth. The proposed approach relies on the coupling of recent contributions presenting: (i) a simulation of temperature in DED, (ii) a mesoscopic model of grain growth model based on Orientated Tessellation Updating Method, and (iii) a macroscopic stochastic model of grain growth. The general strategy is to compute the temperature field as a function of time during the entire process. The initial crystallization is not addressed in this contribution, and an arbitrary initial microstructure are introduced to test the model. The stochastic evolution of the grain structure due to thermal cycling is computed, and the final grain structure statistics is obtained in the entire part. The proposed model is sufficiently fast to enable simulations of large parts and parametric studies or optimization loops can be performed to adjust process parameters.
\end{abstract}

\section{INTRODUCTION}

In this paper, a fast simulation of grain growth during directed energy deposition (DED) is presented. The model is derived for laser metal powder directed energy deposition (LMPDED), although it can be simply adapted for other focused thermal energy (e.g., electron beam, or plasma arc). The analysis of morphological and crystallographic textures obtained in such processes is a major issue in the literature. Indeed, controlling the microstructure is essential to obtain the desired macroscopic behavior. Many studies focus on experimental characterization of the grain structure based on electron back-scatter diffraction techniques (EBSD), which enables to relate process parameters and post-process microstructures. In addition, as the microstructure significantly depends on very local conditions of the melt pool, many papers focus on very detailed simulations of the process, especially the hydrodynamic problem determining the melt pool shape, and cooling and grain growth during crystallization [1-12]. Comprehensive but computationally costly mesoscale simulations include the spreading process of powder, the 
melting phase and grain growth during solidification. However, such comprehensive simulations implies significant computation cost, which is not compatible with macroscopic simulation of the entire process and hinders the development of parametric studies and optimization loops.

In this paper, we present a fast macroscopic simulation of temperature accounting for microstructure evolution (i.e., grain growth). The proposed approach relies on the coupling of recent contributions presenting: (i) a simulation of temperature in DED [13-15], (ii) a mesoscopic model of grain growth model based on Orientated Tessellation Updating Method (OTUM) [16-20], and (iii) a macroscopic stochastic model of grain growth $[17,21]$. The general strategy is to compute the temperature field as a function of time during the entire process. Then, the microstructure after the initial crystallization is estimated from EBSD maps, as this problem is not addressed in this contribution. The stochastic evolution of the grain structure due to thermal cycling is computed for different conditions. The final grain structure statistics is obtained in the entire part. The proposed model is sufficiently fast to enable simulations of large parts and parametric studies or optimization loops can be performed to adjust process parameters.

The paper is organized as follows. The fast thermal analysis coupling heat conduction and phase transitions is broached in section 2. This model should take as inputs the process parameters and material properties and gives as outputs the history of temperature field and phase mixture in the entire part. Then, the fast mesoscopic model of grain growth is briefly presented in section 3. This model takes as inputs the temperature history and the primary microstructure and gives as outputs the secondary microstructure after thermal cycling and grain growth. Since the detailed mesoscopic structure is a rich

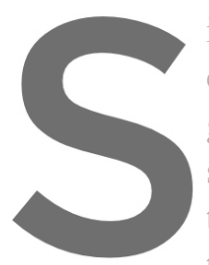
information to be proce of grain growth is also pros grain statistics and provid scale several mesoscopi tics. Thus, the stochastic the mesoscopic scale and he matroscopic seate, ation
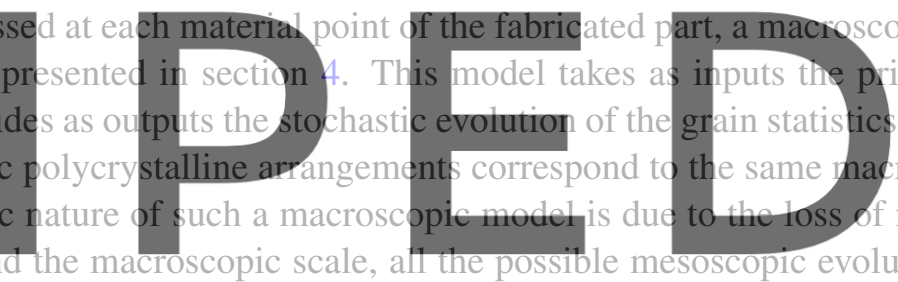
single macroscopic state are considered. The coupling of the different models is broached and tested in

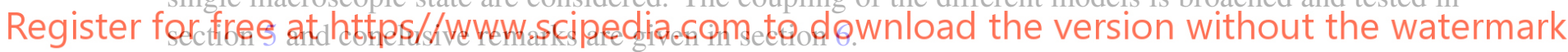

It should be noted that detailed phase transitions are not taken into account in the grain growth model, even though phase proportions are considered in the thermal analysis.

\section{MACROSCOPIC SIMULATION OF TEMPERATURE}

The fast thermal analysis of the entire process [13], which couples heat conduction and phase transitions, enables us to estimate the effect of various process parameters on temperature kinetics and final phase mixture (e.g., for a multiphase steel austenite, ferrite, pearlite, bainite and martensite). In addition, the model enables us to design additional temperature control devices and optimize the process parameters in order to reach a targeted temperature field history and final phase mixture. In addition, during fabrication, the material undergoes volume variations such as thermal expansion and phase transitions, which in turn can be responsible for residual stresses leading to distortion, buckling or failure. Therefore, a fast thermal analysis enables us to design fabrication strategies that minimize residual stresses related to the history of the temperature field. 
The model [13] relies on analytical solutions of the heat equation coupled with numerical procedures to deal with non-linearity of the source associated to phase transitions (liquid to solid and solid to solid). Mathematical developments are not detailed in this paper and the reader is refereed to [13], however, the main assumptions underlying the analytical solution are recalled here for the sake of clarity. The model is adapted for thin-walled structures, and it typically applies to geometries constituted of simple paths in $2 \mathrm{D}$ denoted by $\chi$ and extruded along the vertical direction. The main assumption is to neglect heat fluxes along the tangential direction to the laser path, which is the horizontal direction here. Indeed, excepted in the the very localized melt pool region, heat fluxes along the vertical direction prevail, as shown in the infrared picture presented in figure 1 . The temperature mostly varies along the vertical direction and not along the horizontal direction.

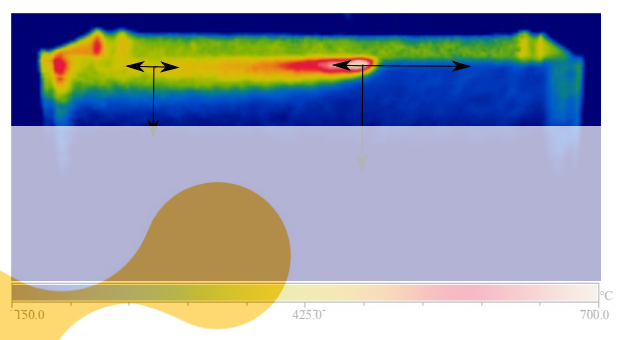

(a) Infrared picture of the process where heat fluxes are schematically represented by arrows

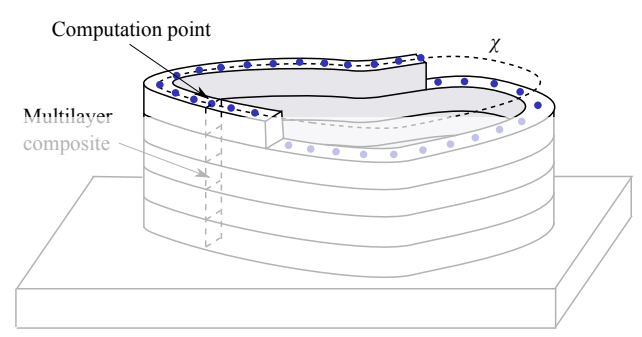

(b) Multilayer composites and computation points
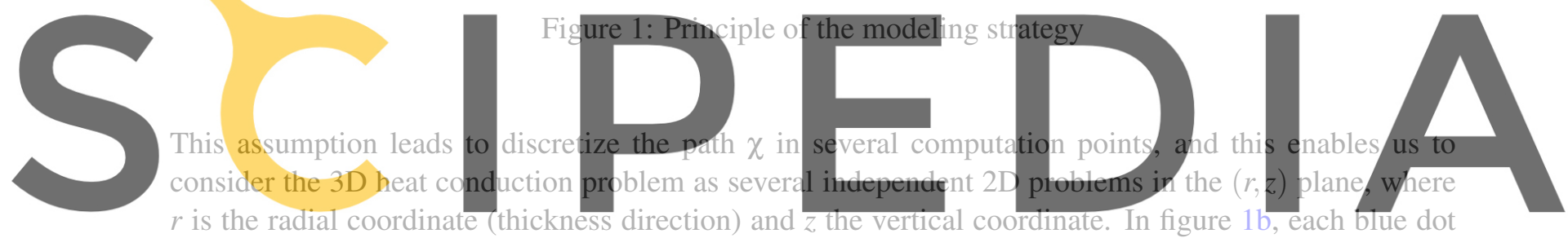

$r$ is the radial coordinate (thickness direction) and $z$ the vertical coordinate. In figure $1 \mathrm{~b}$, each blue dot

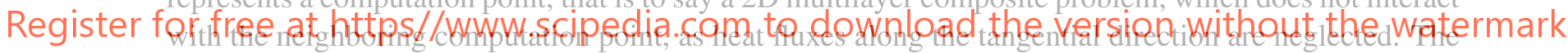

core analytical solution relies on a 2D problem, but pseudo-3D temperature fields can be reconstructed

by combining all the $2 \mathrm{D}$ solutions for all computation points.

In addition, for each computation point, the strategy consists in depositing liquid metal at the deposition temperature, which is an adjustable parameter. Therefore, the powder spray and the melting process are not modeled. The number of layers gradually increases as metal deposition goes on. Each computation point is characterized by its radius of curvature, and the different times when metal is deposited. For each computation point, the simulation strategy consists in approximating the coupled heat equation with phase transitions on each time interval between successive depositions. The first layer is deposited on the substrate and an analytic solution is obtained to compute the temperature history until the next deposition on top of this layer. Then, a second analytic computation is performed to compute the temperature history in the two layers structure until the next deposition. The initial condition of this structure is obtained from the final condition of the previous computation and using the deposition temperature for the top layer. This procedure is repeated until the final number of layers is reached. This strategy is summarized in figure 2 . 
It should be noted that the duration between successive depositions is known from the laser path and process parameters, thus different 3D geometries, laser paths and dwell times can be simulated through different radii of curvature, laser speeds and deposition times.

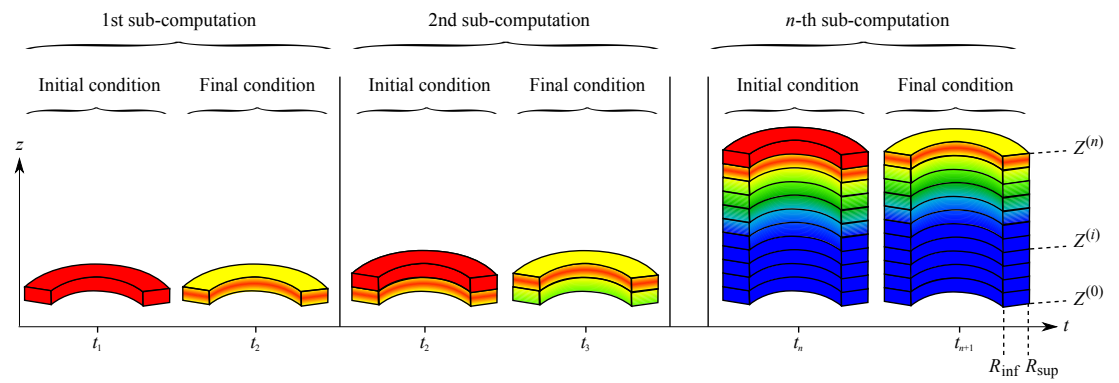

Figure 2: Numerical strategy for each computation point (from [13])

The analytic solution and the numerical coupling with phase transitions obtained with simple Avrami's equations are not detailed in this contribution but the reader is refereed to [13]. However, for each computation point, the $2 \mathrm{D}$ unsteady heat equation with sources in the multilayer composite is recalled here for the sake of clarity.

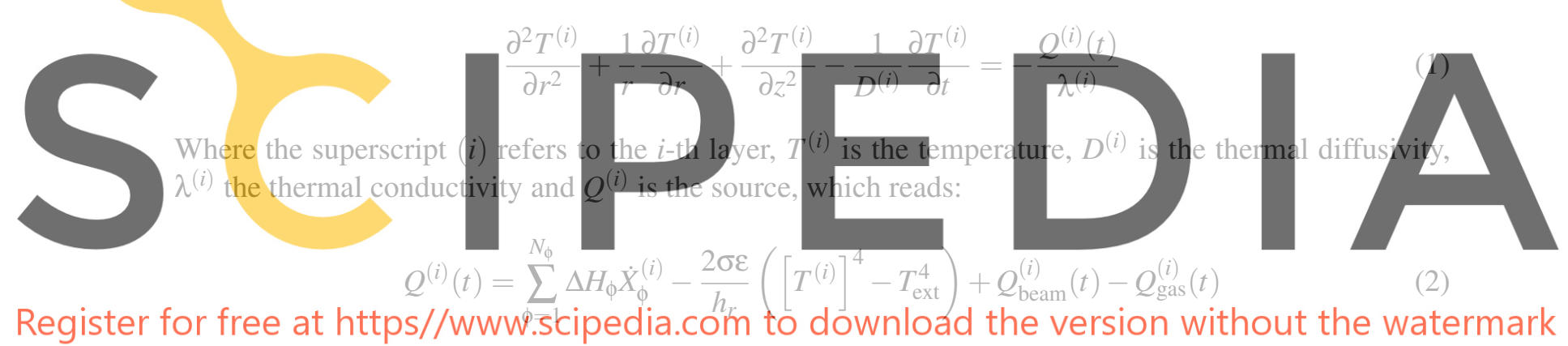

Where $N_{\phi}$ is the number of considered phases (e.g., liquid, austenite, ferrite, etc.), $\Delta H_{\phi}$ is the correspond-

ing latent heat and $\dot{X}_{\phi}^{(i)}$ is the phase proportion rate. In addition, $T_{\mathrm{ext}}$ is the external temperature, $\sigma$ and $\varepsilon$ respectively stand for the Stefan-Boltzmann constant and the emissivity of the surface. The factor $2 / h_{r}$ (where $h_{r}$ denotes the thickness) has been introduced in the radiative term to convert the power at the inner and outer surfaces into a power per unit volume. In addition, $Q_{\text {beam }}^{(i)}$ is the volumetric heat due to the laser, which depends explicitly on the laser speed $V_{\text {beam }}$. Furthermore, $Q_{\text {gas }}^{(i)}(t)$ is the power per unit volume associated to the gas flow carrying the powder, and it corresponds to a convection condition localized around the laser.

For each layer at the inner and outer surfaces, boundary conditions consist in convection conditions with the external temperature and a heat transfer coefficient. In addition, a convection condition at the top surface of the last layer also holds, and a heat resistance between the part and the substrate is introduced. Temperature and heat flux continuity is ensured between layers.

The solution proposed in [13] has been implemented and compared with in-situ measurements performed with infrared pyrometers. 


\section{MESOSCOPIC SIMULATION OF GRAIN GROWTH}

A fast mesoscopic model of grain growth refereed as Orientated Tessellation Updating Method (OTUM) as been proposed in [16]. For the sake of simplicity, the model has been derived for plane hexagonal polycrystals. Misorientation between two neighboring grains (characterized by five parameters in 3D) is characterized only by two parameters in 2D: the misorientation angle (denoted by $\Delta \theta$ ) and the orientation of the grain boundary plane (denoted by $\varphi$ ). This assumption is rather well adapted for thin-walled structure such as those obtained by the DED process. This approach fully relies on Voronoi-Laguerre tessellation techniques that are usually used to approximate polycrystals at the mesoscopic scale (i.e., scale of the polycrystal). Crystal lattice orientation can also be specified for each grain, and the tessellation equipped with such an orientation field is called an Orientated Tessellation (OT). One can approximate the real evolution of the mesostructure as a succession of OT approximations. OTUM relies on the idea that the evolution law of the mesostructure can be formulated directly by modifying the parameters defining the OT. The state variables of the model are therefore not only the temperature but also the parameters defining the OT denoted by $\alpha=(x, y, w, \theta)$ (i.e., seeds coordinates denoted by $(x, y)$, weights denoted by $w$, which define the Voronoi-Laguerre tessellation, and the crystal orientations denoted by $\theta$ ). Therefore there are $4 n$ state variables in the model (where $n$ is the number of grains considered in the polyerystalline structure). However, for the sake of simplicity only the weights $w$ can evolve in this model.

The evolution law of these state variables is formulated at the mesoscopic scale (i.e., for the entire OT). Different energetic contributions are considered so that the evolution law relies on a physically
consistent basis. More precisely, the evolution law is obtained through the energy balance equation at the
mesoscopic scale, by specifying nechanisns at the microscopic scale: (i) the anisotropic grain boundary
(GB) energy, and (ii) the dissipted power through any GB virtual motion. The proposed energetic
framework enables to consider not onty the driving force (associated to the GB energy) but also the
dissipated power as a resistive mechanism. The GB energy is estimated as a function of misorientation

by molecular dynamics computations at the atomic scale. In addition, the dissipated power associated to

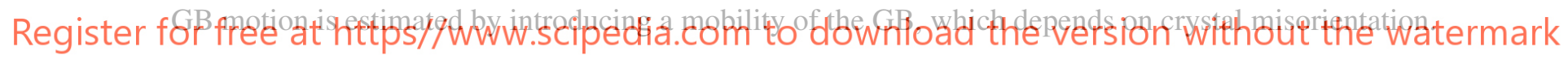
The energetic principle to derive the evolution law for the state variables defining the OT is to compute $\mathcal{E}_{\text {meso }}(T, \alpha)$ the total (sum of all the contributions in the OT) GB energy, and $\mathcal{D}_{\text {meso }}\left(T, \alpha, \dot{w}^{*}\right)$ the total (sum of all the contributions in the OT) dissipated power through any virtual motion of the GBs (where $\dot{w}^{*}$ denotes a virtual evolution rates of the weights). The index meso refers to the fact that mesoscopic quantities are needed to compute the associated variable. As mentioned above, these two functions energetic are obtained from the analysis at the scale of the GB. Then the balance equation is written:

$$
\mathcal{D}_{\text {meso }}(T, \alpha, \dot{w})+\dot{\mathcal{E}}_{\text {meso }}(T, \alpha)=0
$$

The evolution law for the weights $w$ is obtained by maximizing the dissipated power under the constraint of the balance equation (3) and mass conservation, which reads ( $M$ being the mass):

$$
\dot{w}_{\text {meso }}=\left\{\begin{array}{cc}
\underset{w}{\operatorname{argmax}}\left[\mathbb{R}^{n}\left[\mathcal{D}_{\text {meso }}(T, \alpha, \dot{w})\right]\right. \\
\text { subjected to. } & \mathcal{D}_{\text {meso }}(T, \alpha, \dot{w})+\dot{\mathcal{E}}_{\text {meso }}(T, \alpha)=0 \\
\dot{M}=\frac{\partial M}{\partial w} \cdot \dot{w}=0
\end{array}\right.
$$


Analytic computations enables to solve this problem, which in turn can be numerically determined by simply inverting matrices:

$$
\dot{w}_{\text {meso }}=-\frac{m(T)}{L_{0}^{3}} M_{\text {meso }}(\alpha) \cdot \frac{\partial \mathcal{E}_{\text {meso }}(T, \alpha)}{\partial w}
$$

where $w_{\text {meso }}$ are the weights of the OT, $\mathcal{E}_{\text {meso }}$ is the total energy per unit depth in the RVE, $m(T)$ is a scalar mobility $\left(\mathrm{m}^{4} \cdot \mathrm{J}^{-1} \cdot \mathrm{s}^{-1}\right)$ that depends on temperature, and $M_{\text {meso }}$ is a dimensionless mobility second order tensor of size $n \times n$ depending on the mesoscopic state $\alpha=(x, y, w, \theta)$, and obtained analytically. Technicalities are not addressed in this contribution and the reader is refereed to [16] for details. This model has been successfully compared to experimental results on pure iron, but further comparisons with experiments performed on longer durations are still needed.

\section{MACROSCOPIC SIMULATION OF GRAIN GROWTH}

Even though the mesoscopic model introduced in section 3 is relatively fast, a few minutes are necessary to compute the evolution during the fabrication process of a single point in the domain. Therefore a macroscopic stochastic model developed in [21] is also used in this contribution to compute much more rapidly the evolution of grains statistics in the entire domain, instead of the detailed mesostructure.

The macroscopic model relies on state variables that are defined as a statistical description of the grain structure. It is convenient to define dimensionless state variables so that all physical quantities are explicitly written as scaling factors in the dimensionless macroscopic evolution law. State variables are not

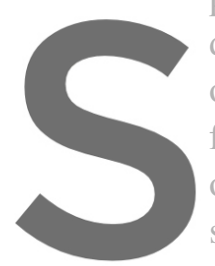
defined arbitrarily as the $m$ of the macroscopic model should face energies carried by th on misorientation, it is statistical distributions of distributions constitute a far too rich information to be reasonably pro

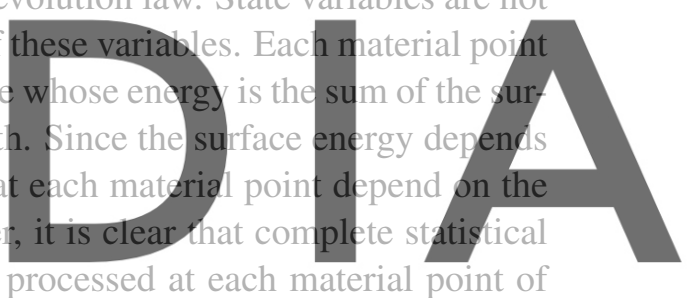

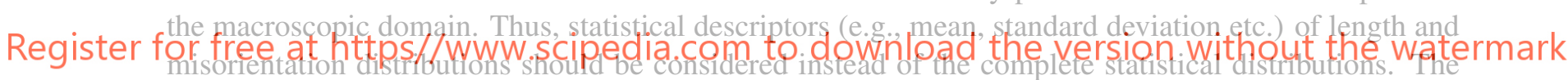
successive statistical moments of the misorientation distribution weighted by the GB lengths are chosen. Since GB lengths continuously tend to zero when a grain disappear, they are used as weights in the statistical moments so that they evolve continuously with respect to time. Thus, the weighted statistical moments obtained from the mesoscopic state read:

$$
\mu_{k}=\sum_{(i, j) \in I} l_{i j} \Delta \theta_{i j}^{k}
$$

where $I$ is the set of grain boundaries defined by the neighboring grains $i$ and $j, \Delta \theta_{i j}$ the misorientations, and $l_{i j}$ the dimensionless GB lengths (physical lengths are obtained by multiplying by a scaling factor $L_{0}(\mathrm{~m})$ ). In addition, $k \in\{0,1,2\}$, which is sufficient to accurately account for the total energy. The statistical moments $\mu_{k}(k \in\{0,1,2\})$ may be computed from the detailed mesoscopic structure by using (6). These variables are denoted by $\mu_{\text {meso }}=\left(\mu_{0}, \mu_{1}, \mu_{2}\right)$ and correspond to a reduction of the amount of data with respect to the complete information needed to characterize the OT. Thus, $\mu_{\text {meso }}$ is a function of $\alpha$ (where $\alpha$ is the mesoscopic state). The vector $\mu_{\text {meso }}$ provides a tool to define macroscopic state variables. Indeed one can demonstrate with a large database of computations at the mesoscopic scale 
(using the model presented in section 3) that the total energy only depends on $\mu_{\text {meso }}$ :

$$
\mathcal{E}_{\text {meso }}(T, \alpha) \approx L_{0} \gamma_{S} \frac{G(T)}{G(0)} f\left(\mu_{\text {meso }}\right)
$$

Where $f\left(\mu_{\text {meso }}\right)$ is a deterministic function identified by using the database of computations at the mesoscopic scale, $\gamma_{S}=1 \mathrm{~J} \cdot \mathrm{m}^{-2}$, and $G(T)$ is the temperature dependent shear modulus that captures the temperature dependance of the GB energy.

From the definition (6), $\mu_{0} / L_{0}$ is the GB length density (length per unit surface). In addition, the mean of misorientations in the RVE denoted by $\widetilde{\mu}_{1}$, and the square of the relative standard deviation, which is the ratio of the standard deviation over the mean, denoted by $\widetilde{\mu}_{2}$ read:

$$
\widetilde{\mu}_{1}=\frac{\mu_{1}}{\mu_{0}} \quad \text { and } \quad \widetilde{\mu}_{2}=\frac{\mu_{2} \mu_{0}}{\mu_{1}^{2}}-1
$$

The evolution law of $\mu_{\text {meso }}$ is obtained from (5) and a simple derivation formula:

$$
\dot{\mu}_{\text {meso }}=-\frac{m(T)}{L_{0}^{3}} \frac{\partial \mu_{\text {meso }}}{\partial w} \cdot M_{\text {meso }} \cdot \frac{\partial \mathcal{E}_{\text {meso }}}{\partial w}
$$

One can demonstrate that there exists a second order mobility tensor $\Gamma_{\text {meso }}(\alpha)$ of size $3 \times 3$ that depends on the entire mesoscopic s
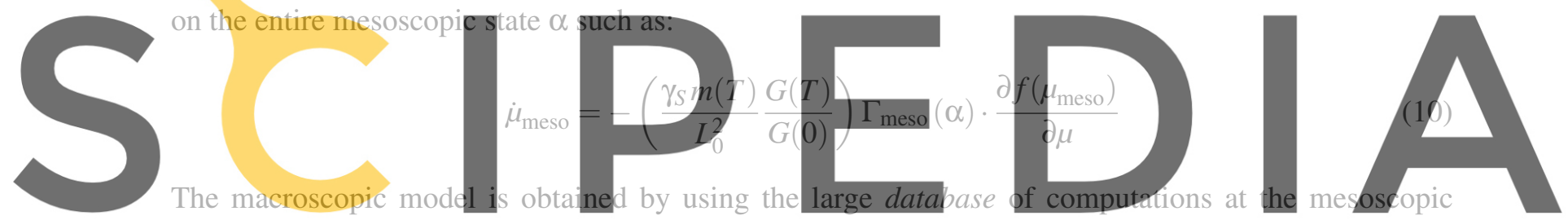

scale to determine a macroscopic second order tensor $\Gamma_{\text {macro }}\left(\mu_{\text {macro }}\right)$ such as $\Gamma_{\text {macro }}\left(\mu_{\text {macro }}\right) \approx \Gamma_{\text {meso }}(\alpha)$,

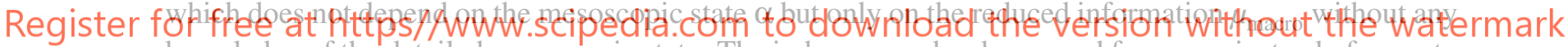
knowledge of the detailed mesoscopic state. The index macro has been used for $\mu_{\text {macro }}$ instead of meso to highlight the fact that the detailed mesoscopic state is unknown. The macroscopic evolution law reads:

$$
\dot{\mu}_{\text {macro }}=-\left(\frac{\gamma_{S} m(T)}{L_{0}^{2}} \frac{G(T)}{G(0)}\right) \Gamma_{\text {macro }}\left(\mu_{\text {macro }}\right) \cdot \frac{\partial f\left(\mu_{\text {macro }}\right)}{\partial \mu}
$$

The mobility tensor $\Gamma_{\text {macro }}\left(\mu_{\text {macro }}\right)$ is determined on the basis of the database of computations at the mesoscopic scale providing numerous estimations of $\Gamma_{\text {meso }}$ associated to $\mu_{\text {meso }}$. The identification is probabilistic as there is an epistemic uncertainty due to the loss of information in the process of reducing the amount of data by introducing the overall state $\mu_{\text {meso }}$. Bayesian inference techniques have been used to estimate the posterior probability density function of $\Gamma_{\text {macro }}\left(\mu_{\text {macro }}\right)$.

For numerical applications, draws of the Bayesian model are used to generate $N_{\text {stat }}=500$ macroscopic evolutions whose means, medians and point-wise standard deviations are computed. This macroscopic model has been compared to various mesoscopic evolutions as introduced in section 3, and a good agreement is observed. 
Table 1: Coefficients for the mobility as a function of temperature

\begin{tabular}{lll}
\hline Molar volume of iron & $V_{m}=7.09 \times 10^{3}$ & $\left(\mathrm{~mm}^{3} \cdot \mathrm{mol}^{-1}\right)$ \\
\hline Grain boundary thickness & $\delta=10^{-6}$ & $(\mathrm{~mm})$ \\
\hline Burger vector & $b^{2}=2.48 \times 10^{-7}$ & $(\mathrm{~mm})$ \\
\hline Universal gaz constant & $\mathrm{R}=8.3144621$ & $\left(\mathrm{~J} \cdot \mathrm{mol}^{1} \cdot \mathrm{K}^{1}\right)$ \\
\hline Activation energy & $Q=139000$ & $\left(\mathrm{~J} \cdot \mathrm{mol}^{-1}\right)$ \\
\hline Diffusivity of Fe atoms & $D_{0}=1.5 \times 10^{2}$ & $\left(\mathrm{~mm}^{2} . \mathrm{s}^{-1}\right)$ \\
\hline
\end{tabular}

\section{RESULTS}

In this section, the thermal model presented in section 2 is used to simulate the evolution of the temperature field during the entire fabrication process for a cylinder. Process parameters are fixed as in [13], and the material is $316 \mathrm{~L}$ stainless steel. Two computations have been performed, the first one allows the substrate to heat up during the fabrication process starting from the room temperature, and the second one assuming that there is a temperature control device associated to the substrate whose temperature is fixed to $800 \mathrm{~K}$. Then, for each material point temperature evolution is used to compute grain growth using both the mesoscopic model presented in section 3 and the macroscopic model presented in section 4 . Even though solidification is taken into account under the form of phase proportion in the thermal model (heat source in the heat equation), there is no solidification model giving the primary microstructure under the form of an OT just after solidification. Of course there are efficient models predicting crys-

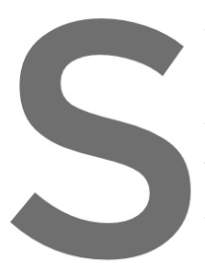
tallization during solidific since this contribution time, a solidification mocel be required, which is still that looks like classical mic
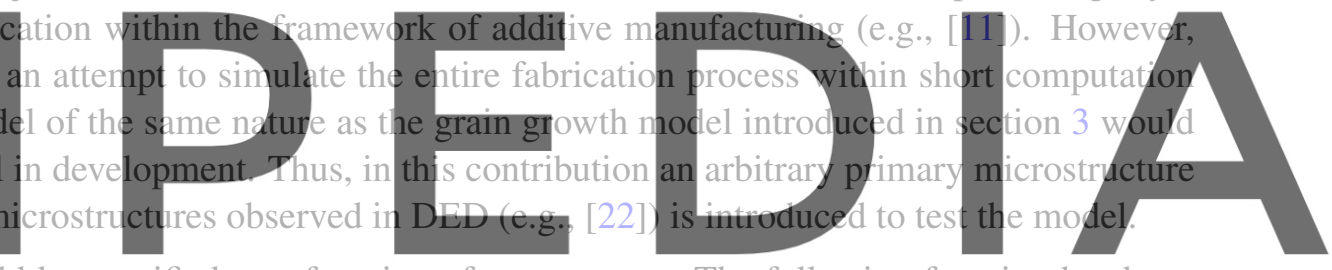

The mobility $m(T)$ should be specified as a function of temperature. The following function has been

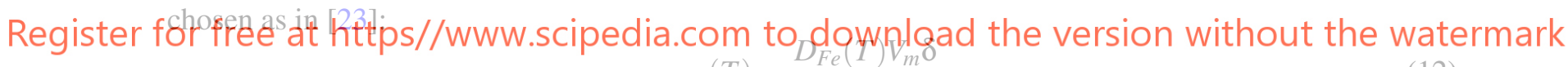
$m(T)=\frac{F e}{b^{2} \mathrm{R} T}$

where $D_{F e}(T)$ follows an Arrhenius law $D_{F e}(T)=D_{0} \exp (-Q / \mathrm{R} T)$, where coefficients are listed in table 1 .

For the first condition (i.e., substrate initially at room temperature), the thermal cycling of one material point is presented in figure 3 with the mesostructure computed with the mesoscopic model (presented in section 3) at some times represented by red dots. It is clear that even though the grain boundary mobility is very significant for high temperatures, the time spent with such high mobility is too short to lead to significant evolution of the mesostructure. For the second condition (i.e., substrate temperature fixed at $800 \mathrm{~K}$ ), results are presented in figure 4. Even though the thermal cycling is similar to the first condition, the overall temperature is bounded by the substrate temperature. Thus, the mobility is sufficiently high during the first temperature peaks to enable rapid evolution the mesostructure during the first few seconds after solidification (i.e., small grains at the inter-layers tend to growth or disappear). Of course, this condition (substrate maintained at high temperature) also favors annealing if the process lasts for several hours. 


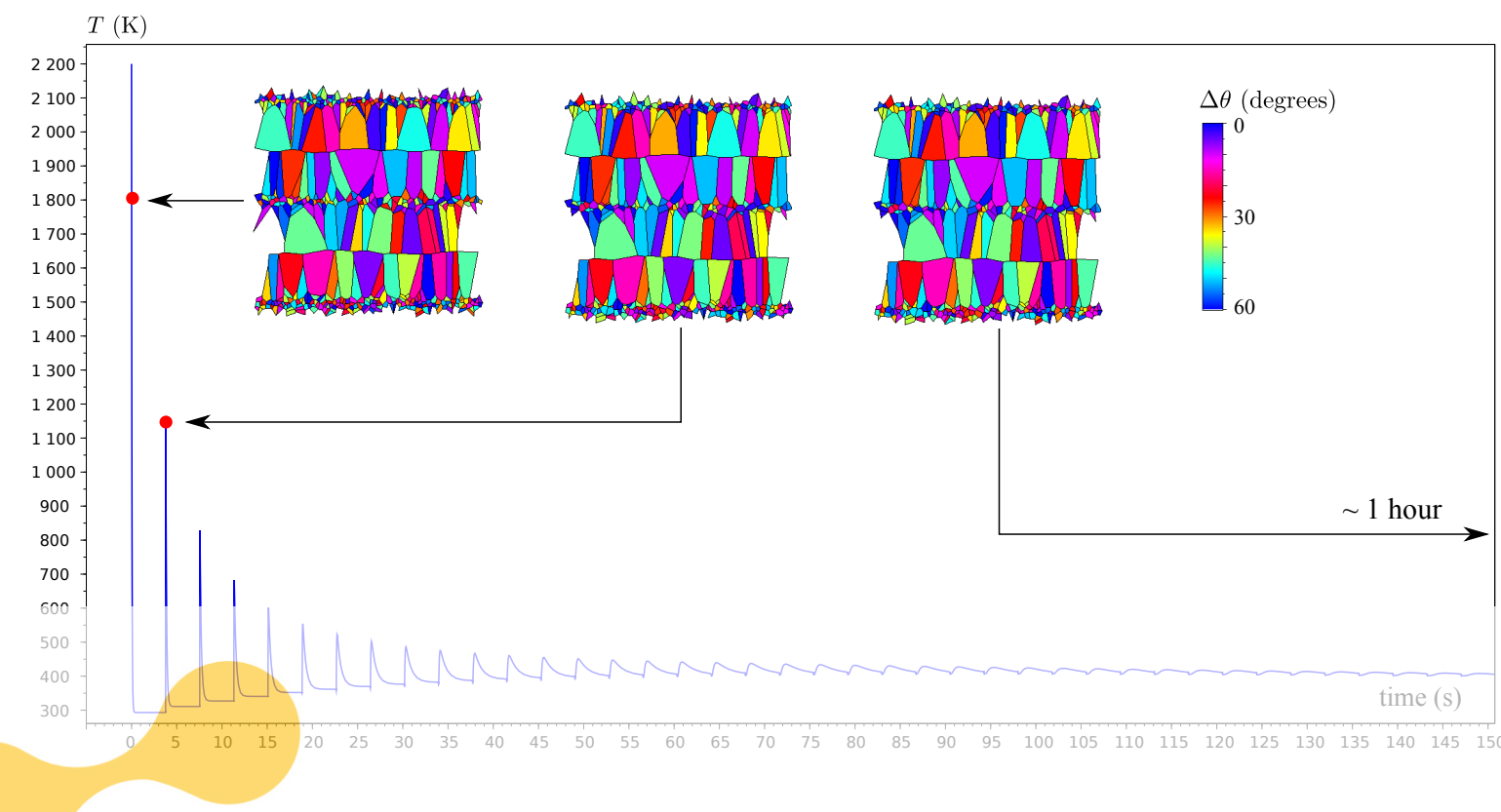

Figure 3: Evolution of the mesostructure, and temperature cycling for the first condition
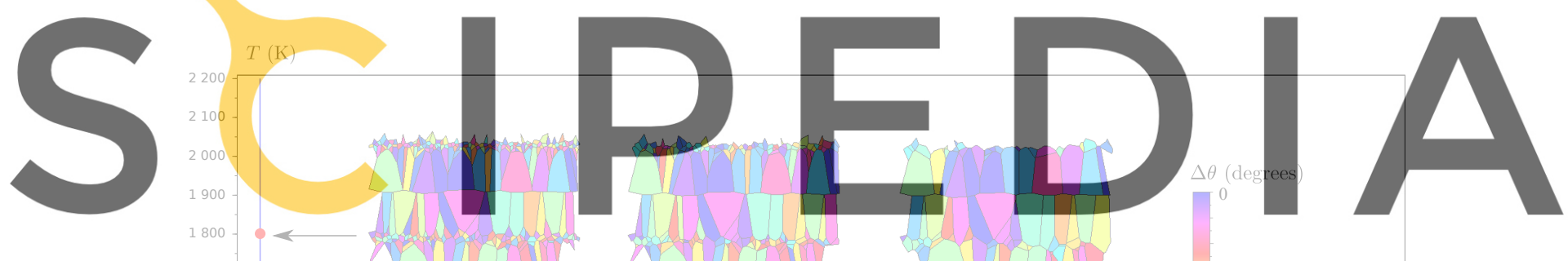

Register for free at https//www.scipedia.com to download the version without the watermark

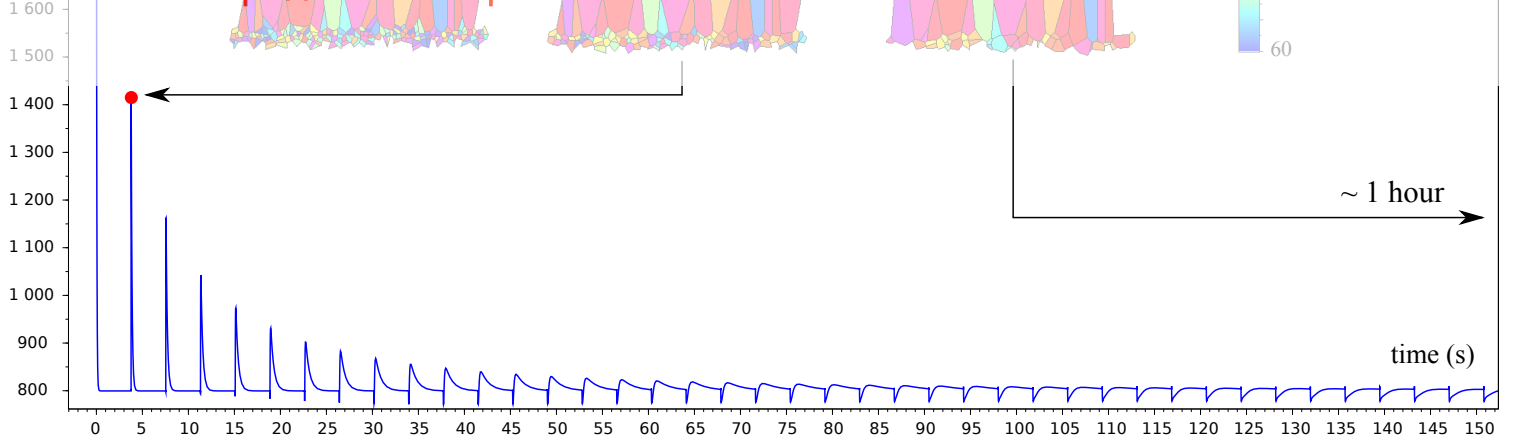

Figure 4: Evolution of the mesostructure, and temperature cycling for the second condition

For the second condition, the evolution of some macroscopic variables $\left(\widetilde{\mu}_{1}\right.$, and $\widetilde{\mu}_{2}$ in (8)) are presented in figures 5 and 6 with the uncertainty given as an envelop representing more or less one standard devi- 
ation. Since this macroscopic model is very fast (only a few seconds for the entire evolution) one can compute the evolution for all material points in the domain, and obtain the history of the fields of the microstructure statistical descriptors with the associated uncertainty. Similarly to the mesoscopic model, the first few temperature peaks are responsible for sudden variation of the grain statistics, and long term annealing condition take place if the process lasts for several hours.

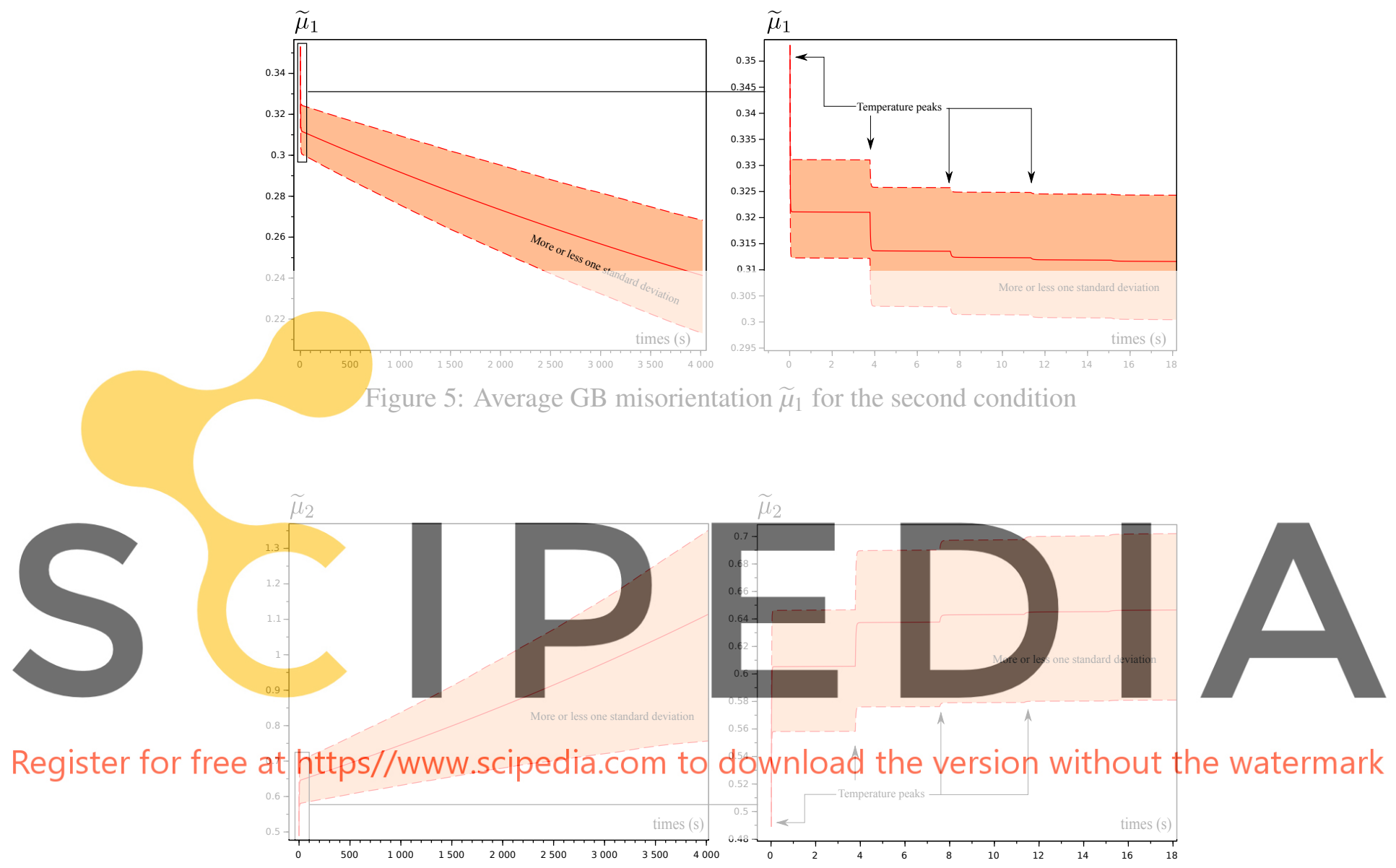

Figure 6: Square of the relative standard deviation of GB misorientation $\widetilde{\mu}_{2}$ for the second condition

\section{CONCLUSION}

This contribution is an attempt to combine several models that have been proposed recently in order to obtain a fast simulation of temperature and grain growth in directed energy deposition at the scale of the entire process. The proposed approach relies on the coupling of recent contributions. The evolution of the grain structure at the mesoscopic scale is possible at few material points during the fabrication process. In addition, the macroscopic statistical descriptors of the microstructure is sufficiently fast to be computed in the entire part during the whole process. Results show that despite very high temperature peaks when the matter is deposited, grain growth does not significantly make evolve the grain structure because of very sharp cooling rates. However, if a temperature control device enables us to maintain the 
substrate temperature at a relatively high value (e.g., $800 \mathrm{~K}$ ), grain growth takes place and the very small grains located at the inter-layers tend to grow or disappear after the first crystallization. In addition, if the process lasts for several hours in this condition, classical annealing conditions take place, which lead to significant evolution of the grain structure.

\section{References}

[1] Xiaoming Zhao, Jing Chen, Xin Lin, and Weidong Huang. Study on microstructure and mechanical properties of laser rapid forming inconel 718. Materials Science and Engineering: A, 478(12):119-124, 2008.

[2] V Manvatkar, A De, and Tarasankar DebRoy. Heat transfer and material flow during laser assisted multi-layer additive manufacturing. Journal of Applied Physics, 116(12):124905, 2014.

[3] William J Sames, FA List, Sreekanth Pannala, Ryan R Dehoff, and Sudarsanam Suresh Babu. The metallurgy and processing science of metal additive manufacturing. International Materials Reviews, 61(5):315-360, 2016.

[4] Denis Carron, Philippe Le Masson, and Rémy Fabbro. 2d longitudinal modeling of heat transfer and fluid flow during multilayered direct laser metal deposition process. JOURNAL (OF LASER APPLICATIONS, 24(3), 2012.

[5] Aref Yadollahi, Nima Shamsaei, Scott M Thompson, and Denver W Seely. Effects of process time interval and heat treatment on the mechanical and microstructural properties of direct laser deposited 3161 stainless steel. Materials Science and Engineering: A, 644:171-183, 2015.

[6] V Manvatkar, A De, and Tarasankar DebRoy. Spatial variation of melt pool geometry, peak temperature and solidification parameters during laser assisted additive manufacturing process. Materials Science and Technology, 31(8):924-930, 2015.

[7] Mustafa Megahed, Hans-Wilfried Mindt, Narcisse NDri, Hongzhi Duan, and Olivier Desmaison. Metal additive-manufacturing process and residual stress modeling. Integrating Materials and Manufacturing Innovation, 5(1):61-93, 2016.

[8] Erdem Kundakcioglu, Ismail Lazoglu, and Suraj Rawal. Transient thermal modeling of laserbased additive manufacturing for $3 \mathrm{~d}$ freeform structures. The International Journal of Advanced Manufacturing Technology, 85(1-4):493-501, 2016.

[9] Yanping Lian, Stephen Lin, Wentao Yan, Wing Kam Liu, and Gregory J Wagner. A parallelized three-dimensional cellular automaton model for grain growth during additive manufacturing. Computational Mechanics, 61(5):543-558, 2018.

[10] Wentao Yan, Stephen Lin, Orion L Kafka, Yanping Lian, Cheng Yu, Zeliang Liu, Jinhui Yan, Sarah Wolff, Hao Wu, Ebot Ndip-Agbor, et al. Data-driven multi-scale multi-physics models to derive process-structure-property relationships for additive manufacturing. Computational Mechanics, 61(5):521-541, 2018. 
[11] HL Wei, GL Knapp, T Mukherjee, and Tarasankar DebRoy. Three-dimensional grain growth during multi-layer printing of a nickel-based alloy inconel 718. Additive Manufacturing, 25:448-459, 2019.

[12] Chamara Kumara, Andreas Segerstark, Fabian Hanning, Nikhil Dixit, Shrikant Joshi, Johan Moverare, and Per Nylén. Microstructure modelling of laser metal powder directed energy deposition of alloy 718. Additive Manufacturing, 25:357-364, 2019.

[13] Daniel Weisz-Patrault. Fast simulation of temperature and phase transitions in directed energy deposition additive manufacturing. Additive Manufacturing, 31:100990, 2020.

[14] Daniel Weisz-Patrault. Fast macroscopic thermal analysis for laser metal deposition. Application to multiphase steels. In Sim-AM 2019: II International Conference on Simulation for Additive Manufacturing, pages 60-71. CIMNE, 2019.

[15] Daniel Weisz-Patrault. Residual strains in directed energy deposition additive manufacturing. In AIP Conference Proceedings, volume 2293, page 020007. AIP Publishing LLC, 2020.

[16] Sofia Sakout, Daniel Weisz-Patrault, and Alain Ehrlacher. Energetic upscaling strategy for grain growth. I: Fast mesoscopic model based on dissipation. Acta Materialia, 196:261-279, 2020.

[17] Sofia Sakout. Energetic upscaling strategy for grain growth. PhD thesis, École des Ponts ParisTech, 2020.

[18] Daniel Weisz-Patrault, Sofia Sakout, and Alain Ehrlacher. Fast simulation of grain growth based on Orientated Tessellation Updating Method. In 24 ème Congrès Français de Mécanique, 2019.

[19] Daniel Weisz-Patrault, Sofia Sakout, and Alain Ehrlacher. Fast simulation of grain growth based on Orientated Tessellation Updating Method. Mechanics \& Industry, 21(5):513, 2020.

[20] Daniel Weisz-Patrault, Sofia Sakout, and Alain Ehrlacher. Fast mesoscopic simulation of grain growth and macroscopic modeling. In AIP Conference Proceedings, volume 2293, page 110007. AIP Publishing LLC, 2020.

[21] Daniel Weisz-Patrault, Sofia Sakout, and Alain Ehrlacher. Energetic upscaling strategy for grain growth. II: Probabilistic macroscopic model identified by Bayesian techniques. Acta Materialia, 2021 (submission).

[22] Yanis Balit, Eric Charkaluk, and Andrei Constantinescu. Digital image correlation for microstructural analysis of deformation pattern in additively manufactured 3161 thin walls. Additive Manufacturing, 31:100862, 2020.

[23] Chad W Sinclair, CR Hutchinson, and Yves Brechet. The effect of nb on the recrystallization and grain growth of ultra-high-purity $\alpha$-fe: a combinatorial approach. Metallurgical and Materials Transactions A, 38(4):821-830, 2007. 